with tamoxifen to induce a fibroblast-specific DOT1L knockout, were injected subcutaneously with bleomycin or vehicle. Injected skin was analyzed by $\mathrm{OH}$-prolin assay and by histology.

Results The DOT1L-inhibitor EPZ-5676 reduced H3K79 dimethylation in all samples. In breast dermal fibroblasts, the induction of ACTA2 with TGF- $\beta$ was reduced with DOT1L inhibition, while in abdominal dermal fibroblasts this induction was more pronounced. After 48 hours of TGF- $\beta$, collagen deposition was higher in DOT1L-inhibited fibroblasts. After 72 hours of TGF- $\beta$ however, this deposition was comparable with controls. DOT1L inhibition induced canonical Wnt signaling in fibroblasts, with a small increase in active $\beta$-catenin and expression of Lymphoid enhancer-binding factor 1 (LEF1). With DOT1L inhibition, more proliferation of fibroblasts, but also proportionally more cells in the G1/G0 phase and less cells in $\mathrm{S}$ and $\mathrm{M} / \mathrm{G} 2$ phase were seen. In vivo, subcutaneous bleomycin increased murine dermal thickness and skin collagen content. No difference was observed between wild type and mice with a fibroblast-specific deletion of DOT1L.

Conclusions In an in vitro model of fibrosis, ACTA2 induction in DOT1L-inhibited human dermal fibroblasts was dependent on the fibroblast origin. DOT1L inhibition resulted in an earlier deposition of collagen, without differences in deposition at the end points. Inhibition of DOT1L induced canonical Wnt signaling and proliferation but also led to a higher proportion of cells in the G0/G1 phase. In an in vivo murine model of skin fibrosis, no difference in bleomycin-induced skin thickness and collagen content was found when the DOT1L gene was deleted in fibroblasts.

Disclosure of Interest None declared.

\begin{tabular}{|l|l}
\hline P111 & TENOCYTES EXTRACTED FROM ROTATOR CUFF \\
TENDONS INDUCE TNAP-DEPENDENT MINERAL \\
DEPOSITION AND EXPRESS GENES RELATED TO A \\
HYPERTROPHIC CHONDROCYTE DIFFERENTIATION
\end{tabular}

${ }^{1} \mathrm{C}$ Darrieutort-Laffite ${ }^{*},{ }^{1} \mathrm{P}$ Arnolfo, ${ }^{1} \mathrm{~A}$ Najm, ${ }^{2} \mathrm{~T}$ Garraud, ${ }^{2} \mathrm{~B}$ Le Goff, ${ }^{1} \mathrm{~F}$ Blanchard. ${ }^{1}$ Inserm UMR1238; ${ }^{2}$ Rheumatology department, CHU Nantes, Nantes, France

10.1136/annrheumdis-2018-EWRR2019.99

Career situation of first and presenting author Resident.

Introduction Calcific tendinopathy represents $10 \%$ to $42 \%$ of chronic painful shoulders. These calcium deposits are composed of carbonated apatite. Although the disease is frequent, its origin stays still largely unknown. Our previous results showed that calcific deposits are surrounded by chondrocytelike cells expressing TNAP (Tissue Nonspecific Alkaline Phosphatase) and ENPP1 (Ectonucleotidepyrophosphatase/phosphodiesterase 1), two key enzymes involved in the mineralization process.

Objectives To study the ability of cells extracted from rotator cuff tendons to produce apatite crystals and to analyze the phenotype of these mineralizing cells.

Methods Tenocytes were extracted from rotator cuff tendons removed during shoulder total replacement. To evaluate their ability to mineralize, they were cultured in an osteogenic medium (OM) for 21 days. Mineral deposition then was assessed by staining with Alizarin red. Tenocytes total RNA was extracted and analyzed by RT-qPCRs. TNAP enzymatic activity was also assessed in the cells. A TNAP inhibitor was used to delineate its implication in the mineralization process.

Results Tendon samples were obtained from 5 patients (age $69.6 \pm 5.13$ years). Cells extracted from these tendons expressed collagen I, collagen III, Scleraxis and Mkx (Mohawk homeobox), as expected for tenocytes. However, Tenomodulin was very weakly expressed and lost after passage 1 . These cells were able to mineralize in the OM although no mineralization was observed in the control medium. qPCR analyses showed a significant increase of TNAP and ENPP1 expression by cells cultured in OM $(\mathrm{p}<0.05)$. Osteoblast markers (Runx2, osteocalcin, osteopontin, BSP) were not increased by the OM. COMP (Cartilage Oligomeric Matrix Protein), a chondrocyte marker was significantly increased, as well as MMP13 (Matrix Metallopeptidase 13) and Collagen $X$ suggesting a hypertrophic differentiation. In parallel, in the OM, TNAP enzymatic activity was significantly higher at 14 and 21 days compared to the control medium. An inhibitor of TNAP completely prevented mineral deposition in OM and reduced expression of the hypertrophic chondrocyte markers MMP13 and Collagen X.

Conclusions Tenocyte-like cells extracted from tendons of the rotator cuff are able to induce mineralization in an osteogenic medium. The cells express genes associated with a hypertrophic chondrocyte phenotype (TNAP, COL10 and MMP13) and TNAP seems to have a crucial role in the induced mineralization. These results suggest that tenocytes could differentiate into hypertrophic chondrocyte which induce TNAP-dependent apatite deposition in calcific tendonitis.

Acknowledgements This study was supported by Inserm and the French Society of Rheumatology.

Disclosure of Interest None declared.

\section{P112 STUDYING CELLULAR SENESCENCE IN HUMAN LYMPH NODE STROMAL CELLS DURING THE EARLIEST PHASES OF RHEUMATOID ARTHRITIS}

1,2 TA De Jong* ${ }^{*}{ }^{1,2} \mathrm{AL}$ Timmerman, 1,2JF Semmelink, 1,2 JS Hähnlein, 1,2 LG van Baarsen. ${ }^{1}$ Department of Rheumatology and Clinical Immunology and Department of Experimental Immunology, Amsterdam Infection and Immunity Institute, Amsterdam UMC, University of Amsterdam; ${ }^{2}$ Academic Medical Center, Amsterdam Rheumatology and Immunology Center (ARC), Amsterdam, Netherlands

\subsection{6/annrheumdis-2018-EWRR2019.100}

Career situation of first and presenting author Student for a master or a $\mathrm{PhD}$.

Introduction Cellular senescence is a state of proliferation arrest of cells. The persistence and accumulation of senescent cells has been implicated in the pathogenesis of age-related diseases. Ageing is an important risk factor of rheumatoid arthritis (RA), a prototypic autoimmune disease in which loss of immune tolerance and systemic autoimmunity precedes clinical onset of disease. Through their intimate contact with lymphocytes, lymph node stromal cells (LNSCs) are important regulators of peripheral tolerance. Therefore, malfunctioning senescent LNSCs may potentially lead to defective peripheral 
tolerance and the development of systemic autoimmune disease.

Objectives To determine the extent of cellular senescence of LNSCs during early phases of systemic autoimmunity.

Methods We included individuals with arthralgia without any evidence of arthritis who were positive for IgM rheumatoid factor (IgM-RF) and/or anti-citrullinated protein antibodies (ACPA; RA-risk group), early arthritis patients (ACR/EULAR 2010 criteria; disease duration $<1$ year) and seronegative healthy controls. All study subjects underwent ultrasoundguided inguinal lymph node biopsy. LNSCs were cultured from freshly collected lymph node needle biopsies and passages $0-9$ were used for experiments. Flow cytometry, qPCR and microscopy were used to measure cell size, granularity, senescence-associated gene expression levels, telomere attrition and senescence-associated $\beta$-galactosidase (SA $\beta$-gal) activity.

Results Preliminary flow cytometry data shows that the cell size of LNSCs from RA patients $(\mathrm{n}=11)$ and RA-risk individuals $(n=7)$ is increased compared with healthy LNSCs $(n=7)$, while granularity was specifically increased in LNSCs from RA patients $(n=9)$. Initial SA $\beta$-gal stainings indicate higher activity in LNSCs from RA-risk $(n=3)$ and RA patients $(n=4)$ compared with healthy controls $(n=2)$, however this data needs to be carefully interpreted and more donors should be analysed. Expression levels of senescence-associated genes significantly increased over culture passages and significantly higher p21 and p53 levels were observed in passage 9 LNSCs from RA patients compared with healthy controls ( $n=6$ per group).

Conclusions These preliminary findings provide a rationale for studying cellular senescence in LNSCs in more detail during different phases of RA and to investigate the consequence of senescent LNSCs on immune cell responses upon their interaction.

Disclosure of Interest None declared.

\section{P113/017 HUMAN LYMPH NODE STROMAL CELLS EXPRESS SELF- ANTIGENS TARGETED BY ANTI-CITRULLINATED PROTEIN ANTYBODIES: ROLE FOR TOLERANCE INDUCTION IN RHEUMATHOID ARTHRITIS}

\begin{abstract}
${ }^{1,2} \mathrm{C}$ Grasso*, 1,2 JS Hähnlein, ${ }^{3} \mathrm{R}$ Nadafi, 1,2TA De Jong, 1,2 $\mathrm{TH}$ Ramwadhdoebe, 1,2JF Semmelink, ${ }^{2} \mathrm{DM}$ Gerlag, ${ }^{2} \mathrm{PP}$ Tak, ${ }^{3} \mathrm{RE}$ Mebius, ${ }^{2} \mathrm{M}$ Safy, ${ }^{4} \mathrm{M}$ Maas, ${ }^{5} \mathrm{Al}$ Catrina, ${ }^{5} \mathrm{H}$ Wähämaa, ${ }^{1,2} \mathrm{LGM}$ Van Baarsen. ${ }^{1}$ Amsterdam Rheumatology and immunology Center (ARC), Academic Medical Center; ${ }^{2}$ Department of Rheumatology and Clinical Immunology, the Department of Experimental Immunology, Amsterdam Infection and Immunity Institute, Amsterdam UMC, University of Amsterdam; ${ }^{3}$ Department of Molecular Cell Biology and Immunology, Amsterdam UMC, Vrije Universiteit Amsterdam,VU medical center; ${ }^{4}$ Depertment of Radiology, Amsterdam UMC, University of Amsterdam, Amsterdam, Netherlands; ${ }^{5}$ Rheumatology Unit, Department of Medicine, Sweden, Karolinska University Hospital and Karolinska Institutet, Stockholm, Sweden
\end{abstract}

\subsection{6/annrheumdis-2018-EWRR2019.101}

Career situation of first and presenting author Student for a master or a PhD.

Introduction In rheumatoid arthritis (RA) the cause for loss of tolerance and anti-citrullinated protein antibody (ACPA) production remains unidentified. Mouse studies showed that peripheral tolerance can be maintained through presentation of peripheral tissue antigens (PTAs) by lymph node stromal cells (LNSCs). We hypothesize that deregulation of peripheral tolerance mechanisms mediated by LNSCs might underlie pathogenesis of RA. Here we investigated the expression of PTAs, citrullinated proteins and immunomodulatory molecules by human LNSCs during health and autoimmunity.

Methods LN tissue sections and LNSCs were prepared from freshly collected lymph node needle biopsies obtained from 24 patients with RA, 23 individuals positive for autoantibodies but without clinical apparent disease (RA-risk group) and 14 seronegative healthy controls. Expression of PADI enzymes, citrullinated proteins, DEAF1, AIRE and PTAs was investigated at mRNA and protein level. Expression of immunomodulatory molecules in LNSCs was assessed after stimulation with IFN $\gamma$ $(\mathrm{n}=15)$.

Results Citrinullated proteins, targeted by ACPA, were found in human LN tissue as well as in cultured LNSCs. In addition, we observed the expression of transcription factors AIRE and DEAF1 as well as disease-related PTAs in LNSCs with some PTAs showing a distinct expression pattern in autoimmune LNSCs compared to healthy controls. TGF- $\beta$ was constitutively expressed by LNSCs while CD86 or IL-10 were not detected. Upon IFN $\gamma$ stimulation LNSCs upregulated MHC class II, co-stimulatory molecules CD40 and CD80 as well as $\mathrm{T}$ cell negative regulators CD274, NOS2 and IDO. Overall, no clear differences between donor groups were observed for these markers with exception of a slightly lower induction of CD40 and NOS2 in RA LNSCs.

Conclusions We present for the first time that human LNSCs express several PTAs and the transcription factors AIRE and DEAF1, driving PTA expression. Additionally, human LNSCs express molecules involved in citrullination, antigen presentation and immunomodulation. Moreover, antigens targeted by ACPAs are present in LN tissue and LNSCs. These data suggest that LNSCs have the machinery to regulate peripheral tolerance.

Disclosure of Interest None declared.

\section{P114 TISSUE REGENERATION AND BONE REPAIR UPON TNF BLOCKADE IN EXPERIMENTAL ARTHRITIS}

K Wanic, on behalf of First author, M Kalkgruber, B Niederreiter, T Shvets, J Smolen, S Hayer*. Medical University of Vienna, Dep Medicine III, Div Rheumatology, Vienna, Austria

\subsection{6/annrheumdis-2018-EWRR2019.102}

Career situation of first and presenting author Student for a master or a $\mathrm{PhD}$.

Introduction Partial bone repair upon treatment with biological DMARDs has been reported in patients with Rheumatoid Arthritis (RA). Previous in vivo PET/CT studies using the human tumor necrosis factor transgenic mouse model (hTNFtg) have been demonstrated the reversibility of preexisting inflammatory, erosive arthritis with complete remission of synovial inflammation and repair of bone erosions upon TNF blockade.

Objectives To investigate spontaneous repair and regenerative processes of structural joint damage in ankle and knee joint, menisci and patella tendon upon TNF blockade in hTNFtg mice.

Methods Two cohorts of arthritic hTNFtg mice were treated with anti-TNF ab (infliximab, $10 \mathrm{mg} / \mathrm{kg}, 3 \mathrm{x}$ per week, i.p., 